\title{
Soliton-antisoliton production in particle collisions
}

\section{Sergei Demidov}

Institute for Nuclear Research of the Russian Academy of Sciences, 60-th October Anniversary pr. 7a, Moscow 117312, Russia

E-mail: demidov@ms2.inr.ac.ru

\section{Dmitry Levkov*}

Institute for Nuclear Research of the Russian Academy of Sciences, 60-th October Anniversary pr. 7a, Moscow 117312, Russia

E-mail: levkov@ms2.inr.ac.ru

We propose general semiclassical method for computing the probability of soliton-antisoliton pair production in collision of two highly energetic particles. Our method is applicable in a class of $(1+1)$-dimensional scalar field theories. We illustrate the method by explicit numerical calculations in a specific model. We find that the probability of the process is exponentially suppressed and remains so at high energies.

The XIXth International Workshop on High Energy Physics and Quantum Field Theory, QFTHEP2010 September 08-15, 2010

Golitsyno, Moscow, Russia

\footnotetext{
*Speaker.
} 
Topological solitons are solutions to the classical field equations whose existence and stability are guaranteed by conservation of topological charge. The simplest soliton is met in $(1+1)-$ dimensional scalar field theory with action

$$
S=\frac{1}{g^{2}} \int d x d t\left(\frac{1}{2}\left(\partial_{\mu} \phi\right)^{2}-V(\phi)\right)
$$

where $g$ is the coupling constant ${ }^{1}$. In what follows we assume weak coupling, $g^{2} \ll 1$. If the potential $V(\phi)$ has two degenerate minima $v_{-}$and $v_{+}$(Fig. 1a, solid line), the model (1) admits soliton and antisoliton solutions interpolating between the minima, see Fig. $1 \mathrm{~b}$.

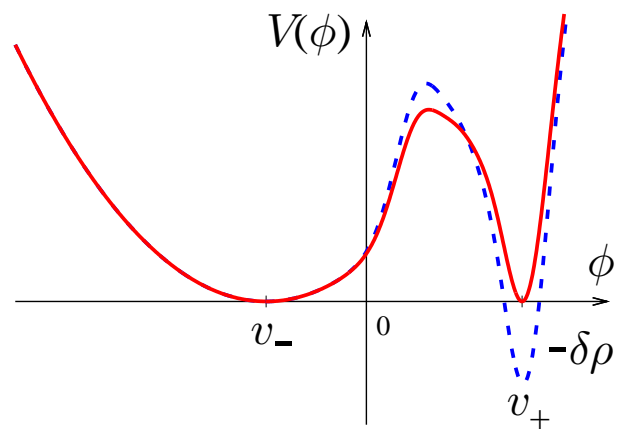

(a)

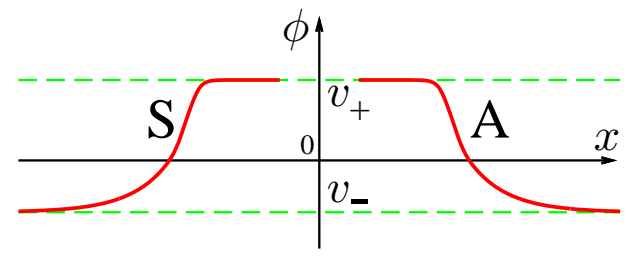

(b)

Figure 1: (a) Potential $V(\phi)$; (b) soliton (S) and antisoliton (A) solutions.

In quantum theory soliton and antisoliton become certain "particle-like" states obeying relativistic energy-momentum relation. In this respect they are similar to perturbative excitations representing quantum particles. Still, at $g^{2} \ll 1$ there is an essential difference between solitons and perturbative excitations. Namely, the size of the (anti)soliton configuration is set by the potential $V(\phi)$ alone, while its mass $M_{S}=M_{A}$ is proportional to the additional factor $1 / g^{2}$, cf. Eq. (1). At the same time, both mass and De Broglie wavelength of perturbative excitations are independent of the coupling constant $g$. Due to this difference, solitons are non-perturbative objects which $\mathrm{can}^{2}$ be described semiclassically while perturbative excitations are not semiclassical.

In this talk we consider inclusive production of soliton-antisoliton pair in collision of two quantum particles. We propose general semiclassical method for calculating the probability of this process. Up to our knowledge, no method of this kind has ever been proposed before, cf. [1].

There are several qualitative arguments in literature [2] stating that the probability of solitonantisoliton pair production in two-particle collisions is exponentially suppressed,

$$
\mathscr{P}(E) \approx A(E) \mathrm{e}^{-F(E) / g^{2}}
$$

where $E \geq 2 M_{S}$ is the energy of the process, $F>0$ is suppression exponent. From the physical viewpoint suppression (2) is due to essential difference in length scales of the initial and final states

\footnotetext{
${ }^{1}$ Substitution $\phi \rightarrow g \phi$ brings $g$ in front of non-linear terms of the potential.

${ }^{2}$ De Broglie wavelength $1 / M_{S}$ of the soliton is much smaller than its size.
} 
of the process. Namely, De Broglie wavelength of two initial particles $1 / E \leq 1 / M_{S}$ is much smaller than the sizes of soliton and antisoliton in the final state.

The exponential form (2) hints that the probability of induced soliton pair production should be calculable semiclassically. However, implementation of the standard semiclassical technique meets two obstacles. First, the initial state of the process is not semiclassical since it contains two quantum particles. Second, soliton and antisoliton attract each other and annihilate classically into $N \sim 1 / g^{2}$ particles. Thus, there is no potential barrier separating soliton-antisoliton pair from the particle sector, and the process itself cannot be treated as potential tunneling.

We solve the first problem by the method of Rubakov, Son and Tinyakov ${ }^{3}$ (RST) [3]. The method is based on the conjecture ${ }^{4}$ that the leading semiclassical exponent $F(E)$ does not depend on the initial-state parameters as long as the latter are not semiclassically large. In particular, $F(E)$ is independent on the number of initial particles $N$ if $N \ll 1 / g^{2}$. RST conjecture is used for calculation of two-particle probability in the following way. Consider inclusive probability of soliton-antisoliton production from multiparticle states,

$$
\mathscr{P}(E, N)=\sum_{i, f}\left|\left\langle f\left|\hat{S} \hat{P}_{E} \hat{P}_{N}\right| i\right\rangle\right|^{2} \approx A(E, N) \mathrm{e}^{-F(E, N) / g^{2}},
$$

where $\hat{S}$ is $S$-matrix, $\hat{P}_{E}$ and $\hat{P}_{N}$ are projectors onto initial states with fixed energy $E$ and multiplicity $N$. Sums in Eq. (3) run over all perturbative initial states above the vacuum $v_{-}$and final states containing soliton-antisoliton pair. At $N \gg 1$ the initial states in Eq. (3) are semiclassical. On the other hand, RST conjecture implies that at $N \ll 1 / g^{2}$ the multiparticle exponent $F(E, N)$ does not depend on $N$ and therefore coincides with the two-particle exponent $F(E)$. In the intersection of two regions $1 \ll N \ll 1 / g^{2}$ semiclassical methods and RST conjecture are both applicable; calculating $F(E, N)$ semiclassically and taking the limit

$$
F(E)=\lim _{g^{2} N \rightarrow 0} F(E, N),
$$

one obtains suppression exponent $F(E)$ of the original two-particle process.

We solve the second problem by introducing potential barrier between the perturbative states and states containing soliton-antisoliton pair. Namely, we modify the potential $V(\phi)$ by adding negative energy density $(-\delta \rho)$ to the vacuum $v_{+}$, see Fig. 1b, dashed line. After modification $v_{-}$and $v_{+}$become false and true vacua respectively, and the process of soliton-antisoliton pair production turns into the process of false vacuum decay. The latter process corresponds to tunneling through the potential barrier [7], with the height of the barrier given by the energy of critical bubble. When $\delta \rho$ goes to zero the critical bubble turns into widely separated soliton-antisoliton pair, and its energy tends to the kinematic threshold $2 M_{S}$ for the soliton-antisoliton pair production.

After the above modifications the probability (3) can be calculated by the standard semiclassical technique. Namely, at small $g^{2}$ the path integral for this probability is saturated by the saddlepoint configuration $\phi(x, t) \in \mathbb{C}$ satisfying the boundary-value problem in Fig. 2 [3]. In particular, $\phi(x, t)$ solves the classical field equations on the contour in complex time plane, where the Euclidean part of the contour corresponds to tunneling. Boundary conditions at $t \rightarrow \pm \infty$ are dictated

\footnotetext{
${ }^{3}$ See Refs. [4] for the alternative method.

${ }^{4}$ This conjecture has been checked in field theory [5] and proved in the context of quantum mechanics [6]. Presently there are no results confronting this conjecture.
} 


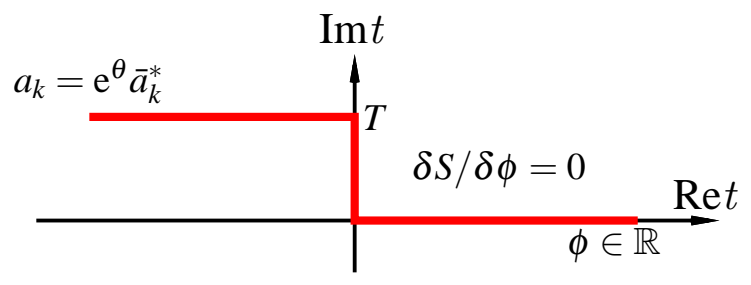

Figure 2: Semiclassical boundary value problem.

by initial and final states of the process: in the asymptotic future solution is real, while at $t \rightarrow-\infty$ its positive and negative frequency components $a_{k}$ and $\bar{a}_{k}$ are linearly related. Parameters $T$ and $\theta$ in Fig. 2 are Lagrange multipliers with respect to energy $E$ and number of particles $N$; in what follows we parametrize solutions with $(E, N)$. After finding solution one computes the multiparticle suppression exponent

$$
F(E, N)=2 g^{2} \operatorname{Im} S[\phi]+\text { boundary terms },
$$

where the boundary terms represent initial- and final-state contributions.

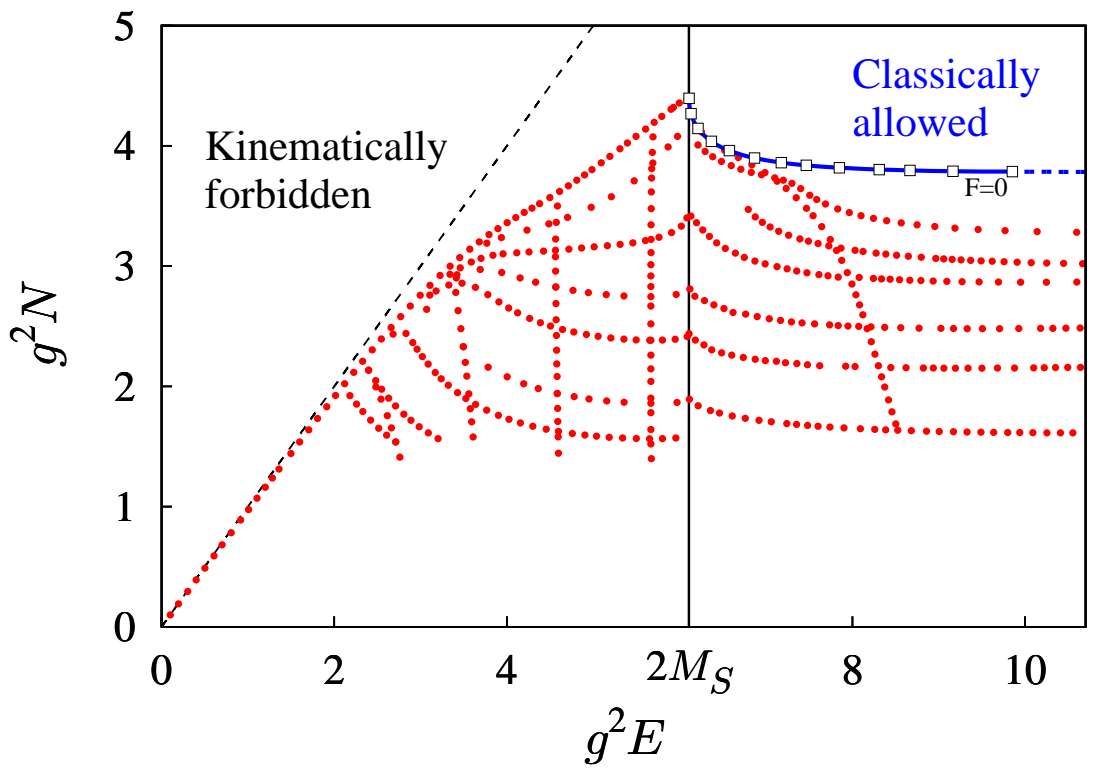

Figure 3: Semiclassical solutions in $E-N$ plane.

To summarize, we formulated a recipe to find the suppression exponent $F(E)$ of solitonantisoliton pair production in two-particle collisions. One starts by describing false vacuum decay in collision of $N \gg 1 / g^{2}$ particles. To this end, one adds energy density $(-\delta \rho)$ to the vacuum $v_{+}$ and solves the boundary value problem in Fig. 2. Substituting semiclassical solution $\phi(x, t)$ into Eq. (5), one computes the multiparticle exponent $F(E, N)$. After that one takes two consecutive limits $g^{2} N \rightarrow 0, \delta \rho \rightarrow 0$ and obtains the sought-for exponent $F(N)$.

We illustrate the method by performing explicit calculations in the potential depicted in Fig. 1a, where dashed and solid lines correspond to the cases $\delta \rho>0$ and $\delta \rho=0$ respectively. We dis- 


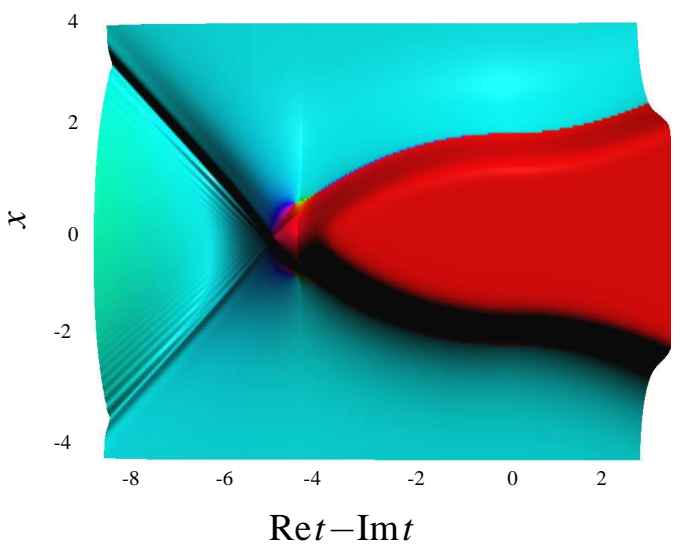

(a)

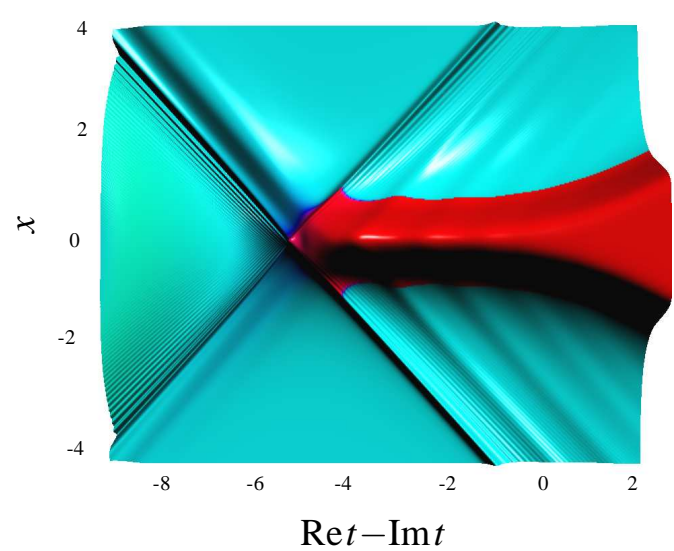

(b)

Figure 4: Semiclassical solutions $\phi(x, t):$ (a) $\left(g^{2} E, g^{2} N\right) \approx(5.0,3.2)$, (b) $\left(g^{2} E, g^{2} N\right) \approx(7.9,2.9)$.

cretize [8] the boundary value problem in Fig. 2 and compute numerically the exponent $F(E, N)$, Eq. (5). We represent numerical solutions by points in $E-N$ plane, see Fig. 3. Solution at $E=N=0$ corresponds to false vacuum decay with no particles in the initial state. This solution is nothing else but bounce [7]; it can be easily found numerically. Solutions with $E, N>0$ are, in fact, distorted bounces. The example of such solution is shown in Fig. 4a. Wave packets in the left part of the figure correspond to particles moving in the initial state; after collision wave packets backreact on the "bounce" part of the solution.

At energies higher than two soliton masses transition mechanism changes [9], cf. [10]. Physically this change is related to the fact that $E=2 M_{S}$ is a kinematic threshold for soliton-antisoliton pair production at $\delta \rho=0$. Thus, semiclassical solutions at $E<2 M_{S}$ and $E>2 M_{S}$ behave differently in the limit $\delta \rho \rightarrow 0$. We observe that solutions at high energies are different, indeed, see Fig. 4b. Visually, these solutions are much smaller and do not change much as $\delta \rho$ decreases.

We extrapolate $F(E, N)$ to $N=0$ and obtain the two-particle exponents $F(E)$ at different $\delta \rho$. The graphs of the latter are plotted in Fig. 5, dashed lines. One sees that the graphs in Fig. 5 have a limit $\delta \rho \rightarrow 0$ (solid line in Fig. 5) corresponding to the exponent $F(E)$.

Conclusion. We formulated the semiclassical method for computing the suppression exponent of soliton-antisoliton pair production in collision of two highly energetic particles. We applied this method to a particular model where the suppression exponent was computed numerically, see Fig. 5, solid line. In this way we explicitly demonstrated that the probability of the process is exponentially suppressed, the suppression exponent being approximately constant at high energies.

Acknowledgements. We thank F.L. Bezrukov, S.M. Sibiryakov and V.A Rubakov for discussions. This work was supported by grants NS-5525.2010.2 (government contact 02.740.11.0244), RFBR-08-02-00473a (S.D.), MK-4317.2009.2 (S.D.), MK-7748.2010.2 (D.L.), fellowship of the "Dynasty" foundation (D.L.) and Russian state contracts $\Pi 520$ (S.D.), П2598 (S.D.). Numerical calculations have been performed on the Computational cluster of the Theoretical division of INR RAS. 


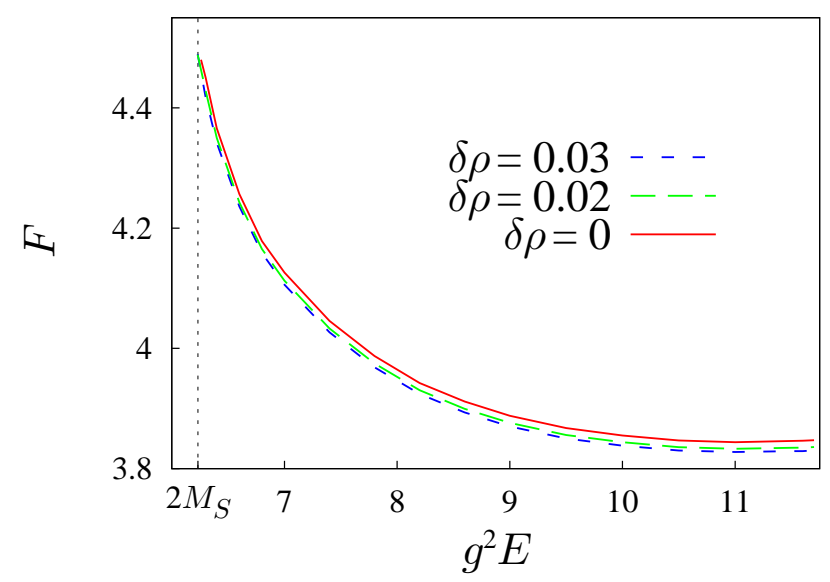

Figure 5: Two-particle suppression exponents $F(E)$ at different $\delta \rho$.

\section{References}

[1] S. Dutta, D.A. Steer and T. Vachaspati, Phys. Rev. Lett. 101, 121601 (2008). T. Romanczukiewicz and Ya. Shnir, Phys. Rev. Lett. 105, 081601 (2010).

[2] A. K. Drukier and S. Nussinov, Phys. Rev. Lett. 49, 102 (1982). R. Singleton Jr., L. Susskind and L. Thorlacius, Nucl. Phys. B 343, 541 (1990). T. Banks, G. Farrar, M. Dine, D. Karabali and B. Sakita, Nucl. Phys. B 347, 581 (1990). V.I. Zakharov, Nucl. Phys. B 353, 683 (1991); Phys. Rev. Lett. 67, 3650 (1991).

[3] V. A. Rubakov, D. T. Son and P. G. Tinyakov, Phys. Lett. B 287, 342 (1992). P. G. Tinyakov, Int. J. Mod. Phys. A 8, 1823 (1993). V.A. Rubakov and M.E. Shaposhnikov, Phys. Usp. 39, 461 (1996) [Usp. Fiz. Nauk 166, 493 (1996)].

[4] S.V. Iordanskii and L.P. Pitaevskii, JETP 49, 386 (1979) [Zh. Eksp. Teor. Fiz. 76, 769 (1979)]. S.Yu. Khlebnikov, Phys. Lett. B 282, 459 (1992). D. Diakonov, V. Petrov, Phys. Rev. D 50, 266 (1994).

[5] P.G. Tinyakov, Phys. Lett. B 284, 410 (1992). A.H. Mueller, Nucl. Phys. B 401, 93 (1993).

[6] G.F. Bonini, A.G. Cohen, C. Rebbi and V.A. Rubakov, Phys. Rev. D 60, 076004 (1999). D.G. Levkov, A.G. Panin, S.M. Sibiryakov, J. Phys. A: Math. Theor. 42, 205102 (2009).

[7] I.Yu. Kobzarev, L.B. Okun, M.B. Voloshin, Sov. J. Nucl.Phys. 20, 644 (1975) [Yad. Fiz. 20, 1229 (1974)]. S.R. Coleman, Phys. Rev. D 15, 2929 (1977) [Erratum-ibid. 16, 1248 (1977)].

[8] A.N. Kuznetsov and P.G. Tinyakov, Phys. Rev. D 56, 1156 (1997). F. Bezrukov, D. Levkov, C. Rebbi, V. Rubakov and P. Tinyakov, Phys. Rev. D 68, 036005 (2003); Phys. Lett. B 574, 75 (2003).

[9] F. L. Bezrukov and D. Levkov, arXiv:quant-ph/0301022; JETP 98, 820 (2004) [Zh. Eksp. Teor. Fiz. 125, 938 (2004)]. D.G. Levkov and S.M. Sibiryakov, Phys. Rev. D 71, 025001 (2005). D.G. Levkov, A.G. Panin and S.M. Sibiryakov, Phys. Rev. Lett. 99, 170407 (2007).

[10] M.B. Voloshin, K.G. Selivanov, Yad. Fiz. 44, 1336 (1986); JETP Lett. 42, 422 (1985) [Pis'ma Zh. Eksp. Teor. Fiz. 42, 342 (1985)]. V.A. Rubakov, D.T. Son and P.G. Tinyakov, Phys. Lett. B 278, 279 (1992). V.G. Kiselev, Phys. Rev. D 45, 2929 (1992). 